\title{
Mutated hilltop inflation revisited
}

\author{
Barun Kumar Pal ${ }^{\mathrm{a}}$ \\ Department of Mathematics, Netaji Nagar College for Women, Kolkata 700 092, India
}

Received: 28 December 2017 / Accepted: 30 April 2018 / Published online: 8 May 2018

(C) The Author(s) 2018

\begin{abstract}
In this work we re-investigate pros and cons of mutated hilltop inflation. Applying Hamilton-Jacobi formalism we solve inflationary dynamics and find that inflation goes on along the $\mathscr{W}_{-1}$ branch of the Lambert function. Depending on the model parameter mutated hilltop model renders two types of inflationary solutions: one corresponds to small inflaton excursion during observable inflation and the other describes large field inflation. The inflationary observables from curvature perturbation are in tune with the current data for a wide range of the model parameter. The small field branch predicts negligible amount of tensor to scalar ratio $r \sim \mathscr{O}\left(10^{-4}\right)$, while the large field sector is capable of generating high amplitude for tensor perturbations, $r \sim \mathscr{O}\left(10^{-1}\right)$. Also, the spectral index is almost independent of the model parameter along with a very small negative amount of scalar running. Finally we find that the mutated hilltop inflation closely resembles the $\alpha$-attractor class of inflationary models in the limit of $\alpha \phi \gg 1$.
\end{abstract}

\section{Introduction}

The standard model of hot Big-Bang scenario is instrumental in explaining the nucleosynthesis, expanding universe along with the formation of cosmic microwave background (CMB henceforth). But there are few limitations in the likes of flatness problem, homogeneity problem etc., which can not be answered within the limit of Big-Bang cosmology. In order to overcome these shortcomings an early phase of accelerated expansion - cosmic inflation was proposed [1-5]. Big-Bang theory is incomplete without inflation and turns into brawny when combined with the paradigm of inflation. Though inflation was initiated to solve the cosmological puzzles, but the most impressive impact of inflation happens to be its ability to provide persuasive mechanism for the origin of cosmological fluctuations observed in the large scale structure and

\footnotetext{
a e-mail: terminatorbarun@gmail.com
}

CMB. Nowadays inflation is the best bet for the origin of primordial perturbations.

Since its inception, almost four decades ago, inflation has remained the most powerful tool to explain the early universe when combined with big-bang scenario. It is still a paradigm due to the elusive nature of the scalar field(s), inflaton, responsible for inflation and the unknown shape of the potential involved. That the potential should be sufficiently flat to render almost scale invariant curvature perturbation along with tensor perturbation [6-12] has been only understood so far. As a result there are many inflationary models in the literature. With the advent of highly precise observational data from various probes [13-16], the window has become thinner, but still allowing numerous models to pass through $[17,18]$. The recent detection of astronomical gravity waves by LIGO $[19,20]$ has made the grudging cosmologists waiting for primordial gravity waves which are believed to be produced during inflation through tensor perturbation. The upcoming stage-IV CMB experiments are expected to constrain the inflationary models further [21] by detecting primordial gravity waves.

The most efficient method for studying inflation is the slow-roll approximation [22,23], where the kinetic energy is assumed to be very small compared to the potential energy. But this is not the only way for successful implementation of inflation and solutions outside slow-roll approximation have been found [24]. In order to study inflationary paradigm irrespective of slow-roll approximation Hamilton-Jacobi formalism $[25,26]$ has turned out to be very handy. Here the inflaton itself is treated as the evolution parameter instead of time, and the Friedmann equation becomes first order which is easy to extract underlying physics from. Another interesting class of inflationary models has been introduced very recently, constant-roll inflation [27-29], where the inflaton rolls at a constant rate.

Here we would like to study single field mutated hilltop model (MHI henceforth) of inflation [30,31] using Hamilton-Jacobi formalism. In MHI observable inflation 
occurs as the scalar field rolls down towards the potential minimum. So MHI does not correspond to usual hilltop inflation [32,33] directly, but the shape of the inflaton potential is somewhat similar to the mutated hilltop in hybrid inflation and hence the name. We shall see that for a wide range of values of the model parameter MHI provides inflationary solution consistent with recent observations. Our analysis also reveals that MHI has two different branches of inflationary solutions: one corresponds to small field inflation and the other represents large field inflation. In earlier studies [30,31] we have reported that MHI can only produce a negligible amount of tensor to scalar ratio, $r \sim 10^{-4}$. But, we shall see here that it is capable of generating $r$ as large as $\mathscr{O}\left(10^{-1}\right)$ depending on the model parameter. Consequently a wide range of $r, 10^{-4} \lesssim r \lesssim 10^{-1}$, can be addressed by MHI. Recent data from Planck [15, 16,34] has reported an upper bound $r_{0.002}<0.07$ and upcoming CMB-S4 experiments are expected to survey tensor to scalar ratio up to $r \sim 2 \times 10^{-3}$ [21]. So sooner or later the model can be tested with the observations. The prediction for inflationary observables from MHI are in tune with recent observations. Further, MHI predicts spectral index which is almost independent of model parameter along with small negative scalar running consistent with current data. It has been also found that MHI closely resembles the $\alpha$ attractor class of inflationary models $[35,36]$ in the limit of $\alpha \phi \gg 1$.

In Sect. 2 we have briefly reviewed Hamilton-Jacobi formalism. In the next Sect. 3 we have discussed about the MHI in Hamilton-Jacobi formalism and have shown resemblance with $\alpha$-Attractor class of inflationary models in Sect. 4. Finally we conclude in Sect. 5 .

\section{Quick look at Hamilton Jacobi formalism}

The Hamilton-Jacobi formalism allows us to recast the Friedmann equation into the following form [24-26,37]

$$
\begin{aligned}
{\left[H^{\prime}(\phi)\right]^{2}-\frac{3}{2 \mathrm{M}_{\mathrm{P}}^{2}} H(\phi)^{2} } & =-\frac{1}{2 \mathrm{M}_{\mathrm{P}}^{4}} V(\phi) \\
\dot{\phi} & =-2 \mathrm{M}_{\mathrm{P}}^{2} H^{\prime}(\phi)
\end{aligned}
$$

where prime and dot denote derivatives with respect to the scalar field $\phi$ and time respectively, and $\mathrm{M}_{\mathrm{P}} \equiv \frac{1}{\sqrt{8 \pi G}}$ is the reduced Planck mass. The associated inflationary potential can then be found by rearranging the terms of Eq. (1)

$V(\phi)=3 \mathrm{M}_{\mathrm{P}}^{2} \mathrm{H}^{2}(\phi)\left[1-\frac{1}{3} \epsilon_{\mathrm{H}}\right]$ where $\epsilon_{\mathrm{H}}$ has been defined as

$\epsilon_{\mathrm{H}}=2 \mathrm{M}_{\mathrm{P}}^{2}\left(\frac{\mathrm{H}^{\prime}(\phi)}{\mathrm{H}(\phi)}\right)^{2}$.

We further have

$\frac{\ddot{a}}{a}=H^{2}(\phi)\left[1-\epsilon_{\mathrm{H}}\right]$

Therefore accelerated expansion takes place when $\epsilon_{\mathrm{H}}<1$ and ends exactly at $\epsilon_{\mathrm{H}}=1$. The evolution of the scale factor turns out to be

$a \propto \exp \left[\int \frac{H}{\dot{\phi}} d \phi\right]$.

The amount of inflation is expressed in terms of number of e-foldings and defined as

$N \equiv \ln \frac{a_{\mathrm{end}}}{a}=\frac{1}{\mathrm{M}_{\mathrm{P}}} \int_{\phi_{\mathrm{end}}}^{\phi} \frac{1}{\sqrt{2 \epsilon_{\mathrm{H}}}} d \phi$.

We have defined $N$ in such a way that at the end of inflation $N=0$ and $N$ increases as we go back in time. The observable parameters are generally evaluated when there are 55-65 efoldings still left before the end of inflation. It is customary to define another parameter by

$\eta_{\mathrm{H}}=2 \mathrm{M}_{\mathrm{P}}^{2} \frac{\mathrm{H}^{\prime \prime}(\phi)}{\mathrm{H}(\phi)}$.

It is worthwhile to mention here that the parameters $\epsilon_{\mathrm{H}}$ and $\eta_{\mathrm{H}}$ are not the usual slow-roll parameters. But in the slowroll limit $\epsilon_{\mathrm{H}} \rightarrow \epsilon$ and $\eta_{\mathrm{H}} \rightarrow \eta-\epsilon$ [23], $\epsilon$ and $\eta$ being usual potential slow-roll parameters.

Though we have not included higher order slow-roll parameters in the present analysis, another widely used higher order slow-roll parameter is defined as follows,

$\zeta_{H}^{2}(\phi) \equiv 4 M_{P}^{4} \frac{H^{\prime}(\phi) H^{\prime \prime \prime}(\phi)}{H^{2}(\phi)}$

In Sect. 3, we shall present variation of the solution of the equation $\zeta_{H}(\phi)=1$ with the model parameter.

\section{Mutated Hilltop inflation: the model}

The potential we would like to study has the following form [30,31]

$V(\phi)=V_{0}[1-\operatorname{sech}(\alpha \phi)]$

where $V_{0}$ is the typical energy scale of inflation and $\alpha$ is a parameter having dimension of inverse Planck mass. The 
potential under consideration does not actually represent typical hilltop inflation $[32,33]$, but the form of the potential is somewhat similar to mutated hilltop inflation in hybrid scenario and hence the name. Accelerated expansion takes place as the inflaton rolls towards the potential minimum. Not only that, from Eq. (10) it is obvious that $V\left(\phi_{\min }\right)=V^{\prime}\left(\phi_{\min }\right)=0$ which is significantly different from the usual hilltop potential.

The associated Hubble parameter may be written as

$H(\phi) \simeq \sqrt{\frac{V_{0}}{3 M_{\mathrm{P}}^{2}}}[1-\operatorname{sech}(\alpha \phi)]^{\frac{1}{2}}$

The value of the constants can be fixed from the conditions for successful inflation and the observational bounds.

The parameters $\epsilon_{H}, \eta_{H}, \zeta_{H}$ in the Hamilton-Jacobi formalism now take the form

$$
\begin{aligned}
\epsilon_{H} \simeq & \frac{\mathrm{M}_{\mathrm{P}}^{2} \alpha^{2}}{2} \frac{\operatorname{sech}^{2}(\alpha \phi) \tanh ^{2}(\alpha \phi)}{(1-\operatorname{sech}(\alpha \phi))^{2}} \\
\eta_{H} \simeq & -\frac{\mathrm{M}_{\mathrm{P}}^{2} \alpha^{2}}{2} \operatorname{sech}(\alpha \phi)[2+3 \operatorname{sech}(\alpha \phi)] \\
\zeta_{H} \simeq & \frac{M_{P}^{2}}{2} \\
& \times \sqrt{\alpha^{4}(6 \cosh (\alpha \phi)+2 \cosh (2 \alpha \phi)-13) \operatorname{coth}^{2}(\alpha \phi / 2) \operatorname{sech}^{4}(\alpha \phi)}
\end{aligned}
$$

In Fig. 1 we have plotted the solutions of $\epsilon_{H}=1$ and $\left|\eta_{H}(\phi)\right|=1$ obtained by solving the background evolution numerically along with those found using the approximate form Eq. (11) for the Hubble parameter. The background evolution was found numerically by solving Eq. (1) with the potential given by Eq. (10). From the figure it is clear that $\left|\eta_{H}\right|=1$ occurs well before $\epsilon_{H}=1$ for $\alpha \geq \alpha_{\text {eq }}$ in both the cases, where $\alpha_{\text {eq }}$ represents the value of $\alpha$ for the simultaneous occurrence of $\left|\eta_{H}\right|=1$ and $\epsilon_{H}=1$ (values of $\alpha_{\text {eq }}$ are different in exact and approximate solutions).

In the Hamilton-Jacobi formalism with Hubble slow-roll parameters the end of inflation is explicitly given by $\epsilon_{H}=1$ at $\phi_{\text {end }}[23,24]$, which is also clear from Eq. (5). From the Fig. 1 it may be seen that the exact numerical solution of $\epsilon_{H}=1$ (the solid red line) is close to that obtained by using the approximated form of the Hubble parameter (dashed magenta line), and differs slightly for $\left|\eta_{H}\right|=1$. But as we are using an approximated form of the Hubble parameter, we shall consider the end of inflation to be where slow-roll approximation breaks, i.e. $\max _{\phi}\left\{\epsilon_{H}=1,\left|\eta_{H}\right|=1\right\}$. Now the solution of $\epsilon_{H}=1$ is the root of the following equation

$\mathrm{M}_{\mathrm{P}}^{2} \alpha^{2} \operatorname{sech}^{2}\left(\alpha \phi_{\epsilon}\right)\left(1-\operatorname{sech}^{2}\left(\alpha \phi_{\epsilon}\right)\right) \simeq 2\left(1-\operatorname{sech}\left(\alpha \phi_{\epsilon}\right)\right)^{2}$.

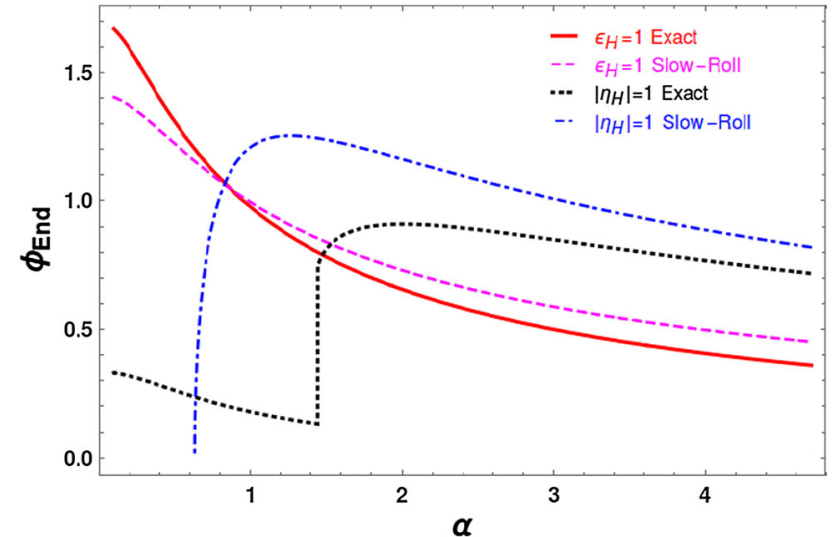

Fig. 1 The solid red line is the solution of $\epsilon_{H}=1$ obtained numerically from the background evolution and the dashed magenta line is the solution of $\epsilon_{H}=1$ using slow-roll approximation. The black dotted line is the solution of $\left|\eta_{H}\right|=1$ obtained numerically from the background evolution and the dot-dashed blue line is the solution of $\left|\eta_{H}\right|=1$ in the slow-roll approximation

Equation (13) can be solved analytically and the relevant solution turns out to be

$$
\begin{array}{r}
\phi_{\epsilon} \simeq \mathrm{M}_{\mathrm{P}} b^{-1} \operatorname{sech}^{-1} \frac{1}{3} \\
\times\left[\begin{array}{c}
b^{2}-6 \\
-1+\frac{\left(36 b-b^{3}+3 \sqrt{6} \sqrt{4+22 b^{2}-b^{4}}\right)^{1 / 3}}{b\left(36 b-b^{3}+3 \sqrt{6} \sqrt{4+22 b^{2}-b^{4}}\right)^{1 / 3}} \\
+
\end{array}\right]
\end{array}
$$

where $b \equiv \alpha \mathrm{M}_{\mathrm{P}}$. On the other hand the solution of $\left|\eta_{H}\right|=1$ is given by

$\phi_{\eta} \simeq \alpha^{-1} \operatorname{sech}^{-1} \frac{1}{3}\left[-1+\sqrt{1+\frac{6}{b^{2}}}\right]$.

So the end of inflation may be written as $\phi_{\text {end }} \simeq \max \left\{\phi_{\epsilon}, \phi_{\eta}\right\}$, i.e. $\phi_{\mathrm{end}} \simeq \phi_{\epsilon}$ and $\phi_{\mathrm{end}} \simeq \phi_{\eta}$ for $\alpha \leq \alpha_{\mathrm{eq}}$ and $\alpha>\alpha_{\mathrm{eq}}$ respectively.

In Fig.2 we have plotted the solutions of $\epsilon_{\mathrm{H}}=1,\left|\eta_{\mathrm{H}}\right|=1$ and $\left|\zeta_{H}\right|=1$ using the Hubble parameter as given by Eq. (11). From the figure we see that $\left|\eta_{H}\right|$ becomes order of unity before $\epsilon_{\mathrm{H}}$, but $\left|\zeta_{H}\right|$ remains small.

From now on all the results that we shall present in this article are based on the approximated form of the Hubble parameter and without considering the effect of higher order slow-roll parameters. 


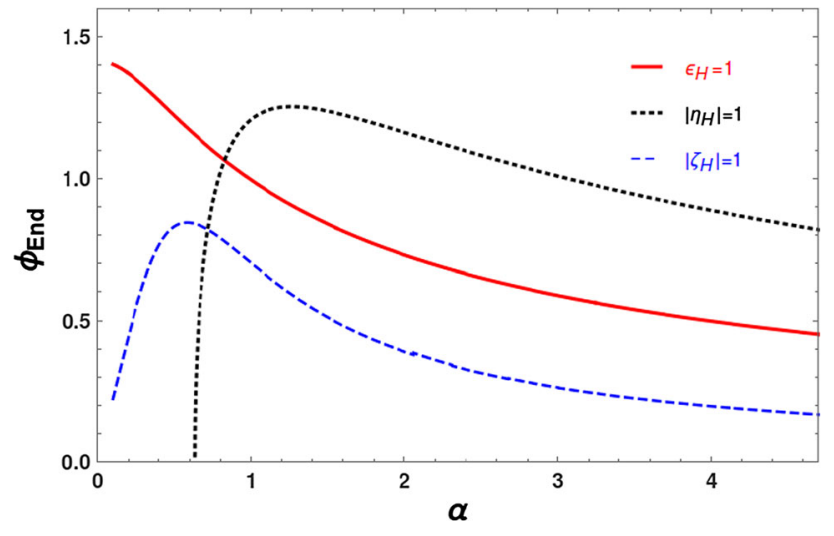

Fig. 2 The solutions of $\epsilon_{\mathrm{H}}=1$ (solid red line), $\left|\eta_{\mathrm{H}}\right|=1$ (dotted black line) and $\left|\zeta_{H}\right|=1$ (dashed blue line) have been plotted. The approximated form of the Hubble parameter has been used to find these solutions

\subsection{Number of e-foldings}

The number of e-foldings in MHI is found to have the following form

$$
\begin{aligned}
& N(\phi) \simeq \frac{1}{\alpha^{2} \mathrm{M}_{\mathrm{P}}^{2}} \\
& \quad \times\left[\cosh (\alpha \phi)-\cosh \left(\alpha \phi_{\mathrm{end}}\right)-2 \ln \frac{\cosh (\alpha \phi / 2)}{\cosh \left(\alpha \phi_{\text {end }} / 2\right)}\right] .
\end{aligned}
$$

The above Eq. (16) can be analytically inverted to get the scalar field as a function of e-foldings as follows

$$
\begin{aligned}
\phi \simeq & \alpha^{-1} \cosh ^{-1}\left[-1-\mathscr{W}_{-1}\right. \\
& \left.\times\left(-\left[\cosh \left(\alpha \phi_{\text {end }}\right)+1\right] e^{-\mathrm{M}_{\mathrm{P}}^{2} \alpha^{2} \mathrm{~N}-1-\cosh \left(\alpha \phi_{\text {end }}\right)}\right)\right] \\
= & \alpha^{-1} \cosh ^{-1}(\mathscr{L} W[\alpha, N])
\end{aligned}
$$

where we have defined $\mathscr{L} W[\alpha, N] \equiv-1-\mathscr{W}_{-1}$ $\left(-\left[\cosh \left(\alpha \phi_{\text {end }}\right)+1\right] e^{-\mathrm{M}_{\mathrm{P}}^{2} \alpha^{2} \mathrm{~N}-1-\cosh \left(\alpha \phi_{\text {end }}\right)}\right)$ and $\mathscr{W}_{-1}$ is the Lambert function. From the above Eq. (17), one can see that mutated hilltop inflation occurs along the $\mathscr{W}_{-1}$ branch of the Lambert function, first pointed out in Ref. [17]. The value of the inflaton when cosmological scale leaves the horizon, $\phi_{\mathrm{CMB}}$, is then given by

$\phi_{\mathrm{CMB}} \simeq \alpha^{-1} \cosh ^{-1}\left(\mathscr{L} W\left[\alpha, N_{\mathrm{CMB}}\right]\right)$

The slow-roll parameters now can be expressed as a function of the e-foldings

$\epsilon_{H}=\frac{1}{2 \mathrm{M}_{\mathrm{P}}^{2}}\left(\frac{d \phi}{d N}\right)^{2}$

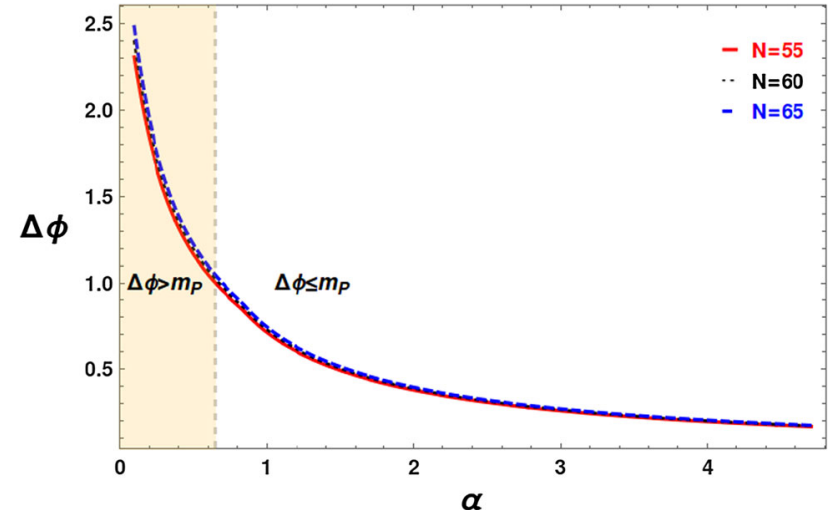

Fig. 3 Excursion of the scalar field (in the unit of $m_{p}$ ) with the model parameter $\alpha$ for three different values of e-foldings, $N=55,60,65$. The dotted vertical line corresponds to the value of $\alpha$ at which $\Delta \phi=m_{P}$ which has been estimated by considering $N_{\mathrm{CMB}}=55$

$$
\begin{aligned}
& \simeq \frac{\mathrm{M}_{\mathrm{P}}^{2} \alpha^{2}}{2} \frac{\mathscr{L} W[\alpha, N]+1}{(\mathscr{L} W[\alpha, N]-1) \mathscr{L} W[\alpha, N]^{2}} \\
\eta_{H} & =\left(\frac{d^{2} \phi}{d N^{2}}\right)\left(\frac{d \phi}{d N}\right)^{-1}+\epsilon_{H} \\
& \simeq-\frac{\mathrm{M}_{\mathrm{P}}^{2} \alpha^{2}}{2} \frac{2 \mathscr{L} W[\alpha, N]+3}{\mathscr{L} W[\alpha, N]^{2}}
\end{aligned}
$$

This makes life simpler as now all the inflationary observable parameters when derived in the slow-roll limit can be expressed as a function of $N$.

\subsection{The Lyth bound for MHI}

The fluctuations in the tensor modes solely depends on the Hubble parameter whereas curvature perturbation is a function of the Hubble parameter and inflaton. Consequently, tensor to scalar ratio determines excursion of the inflaton during observable inflation, first shown in Ref. [38] and known as Lyth bound

$\Delta \phi=\frac{\mathrm{m}_{\mathrm{P}}}{8 \sqrt{\pi}} \int_{0}^{N_{\mathrm{CMB}}} \sqrt{r} d N$

where $\mathrm{m}_{\mathrm{P}}=2 \sqrt{2 \pi} \mathrm{M}_{\mathrm{P}}$ is the actual Planck mass. $\Delta \phi \geq \mathrm{m}_{\mathrm{P}}$ corresponds to large field model and $\Delta \phi<\mathrm{m}_{\mathrm{P}}$ small field models. One expects to get larger tensor to scalar ratio, $r$, where $\Delta \phi \geq \mathrm{m}_{\mathrm{P}}$ due to the higher energy scale required for successfully explaining the observable parameters. For the model under consideration we have found

$\Delta \phi \simeq \alpha^{-1} \cosh ^{-1}\left(\mathscr{L} W\left[\alpha, N_{\mathrm{CMB}}\right]\right)-\alpha^{-1} \cosh ^{-1}(\mathscr{L} W[\alpha, 0])$

In Fig. 3 we have shown the variation of the scalar field excursion in the unit of $\mathrm{m}_{\mathrm{P}}$, with the model parameter $\alpha$. 


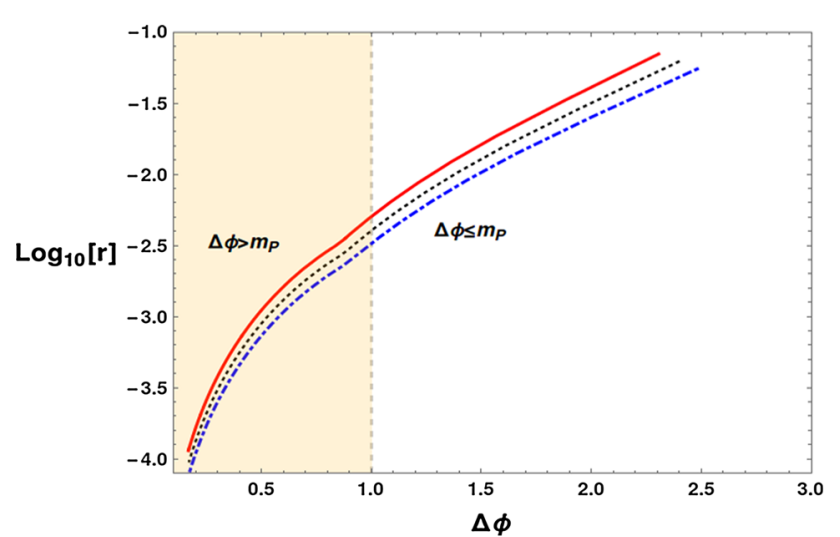

Fig. 4 The logarithmic variation of the tensor to scalar ratio with $\Delta \phi$ for three values of $N_{\mathrm{CMB}}$ has been plotted. Red solid line for $N_{\mathrm{CMB}}=$ 55 , black dotted curve represents $N_{\mathrm{CMB}}=60$ and the blue dashed line for $N_{\mathrm{CMB}}=65$

From the figure it is obvious that the mutated hilltop model of inflation has small excursion of the inflaton for $\alpha \geq \alpha_{\Delta \phi=1}$ and large field excursion for $\alpha<\alpha_{\Delta \phi=1}$, where $\alpha_{\Delta \phi=1}$ is the solution of Eq. (22) for $\alpha$ with $\Delta \phi=\mathrm{m}_{\mathrm{P}}$. So this model is capable of addressing both the large and small field inflationary scenarios for suitable values of the model parameter.

In Fig. 4 we have shown the variation of $\log _{10} \mathrm{r}$ with $\Delta \phi$. From the figure we see that small field MHI may give rise to negligible amount of tensor to scalar ratio, $r \sim \mathscr{O}\left(10^{-4}\right)$, on the other hand for large $\Delta \phi, r$ can be as large as $\mathscr{O}\left(10^{-1}\right)$.

\subsection{Inflationary observables in the slow-roll limit}

The inflationary observable parameters can be expressed in terms of the slow-roll parameters. The power spectrum of the curvature perturbation turns out to be

$$
\begin{aligned}
P_{\mathscr{R}} & \simeq \frac{1}{16 \pi^{2} \mathrm{M}_{\mathrm{P}}^{4}}\left[\frac{H(\phi)^{2}}{H^{\prime}(\phi)}\right]_{\phi=\phi_{\mathrm{CMB}}}^{2} \\
& \simeq \frac{V_{0}}{12 \pi^{2} \alpha^{2} \mathrm{M}_{\mathrm{P}}^{6}} \frac{\mathscr{L} W\left[\alpha, N_{\mathrm{CMB}}\right]\left(\mathscr{L} W\left[\alpha, N_{\mathrm{CMB}}\right]-1\right)^{2}}{\mathscr{L} W\left[\alpha, N_{\mathrm{CMB}}\right]+1}
\end{aligned}
$$

In Fig. 5 we have shown the variation of the typical energy scale associated with MHI for different values of the model parameter. From the figure it is clear that maximum energy scale that can be achieved is $\mathscr{O}\left(10^{16}\right) \mathrm{GeV}$. To determine this energy scale we have used $P_{\mathscr{R}}=2.142 \times 10^{-9}$ from Planck 2015 result [16]. The scale dependence of the spectrum of curvature perturbation is described by spectral index. In MHI we have found

$$
\begin{aligned}
n_{S} & \simeq 1-\left.4 \epsilon_{H}\right|_{\phi=\phi_{\mathrm{CMB}}}+\left.2 \eta_{H}\right|_{\phi=\phi_{\mathrm{CMB}}} \\
& \simeq 1-\mathrm{M}_{\mathrm{P}}^{2} \alpha^{2} \frac{2 \mathscr{L} \mathrm{W}\left[\alpha, \mathrm{N}_{\mathrm{CMB}}\right]^{2}+3 \mathscr{L} \mathrm{W}\left[\alpha, \mathrm{N}_{\mathrm{CMB}}\right]-1}{\mathscr{L} \mathrm{W}\left[\alpha, \mathrm{N}_{\mathrm{CMB}}\right]^{2}\left(\mathscr{L} \mathrm{W}\left[\alpha, \mathrm{N}_{\mathrm{CMB}}\right]-1\right)}
\end{aligned}
$$

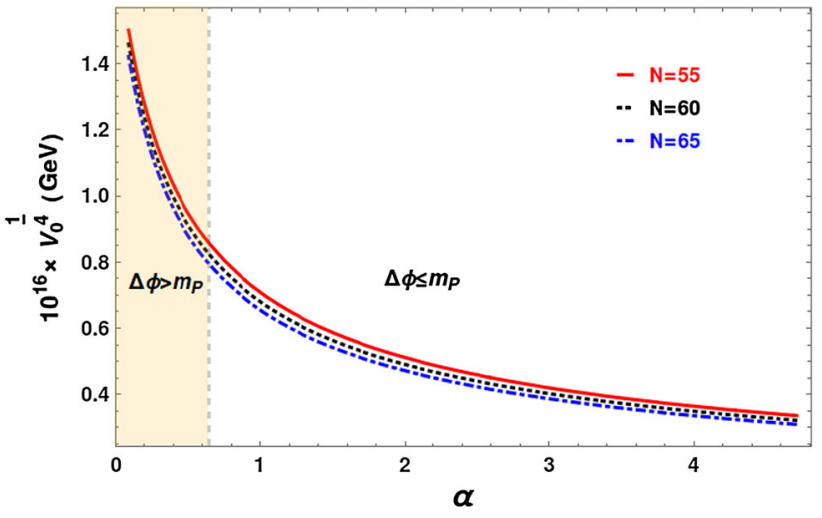

Fig. 5 The energy scale in the unit of GeV for MHI has been plotted with the model parameter $\alpha$ for $N_{\mathrm{CMB}}=55,60,65$. The shaded region corresponds to the large field sector for MHI

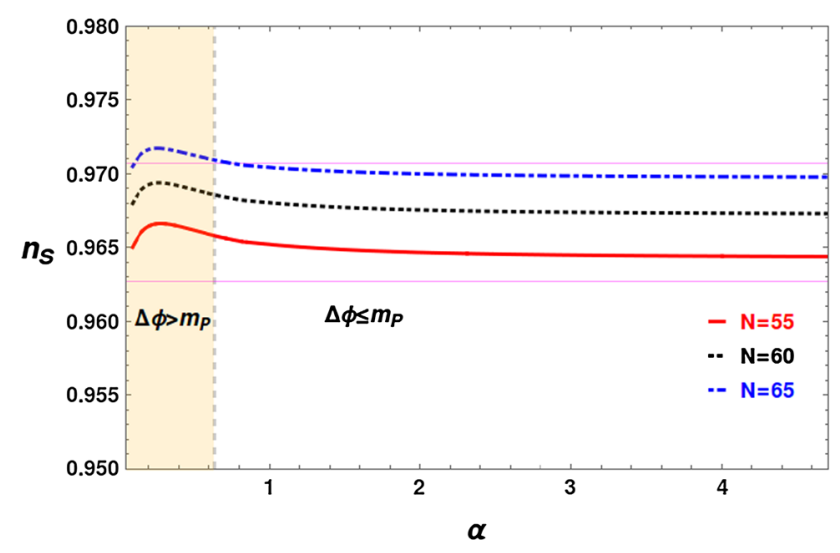

Fig. 6 The variation of the scalar spectral index with $\alpha$ for three different values of $N_{\mathrm{CMB}}$ has been plotted. The shaded vertical region is the result for large field sector of MHI. The two horizontal lines (magenta) are for Planck 2015 upper and lower bounds on $n_{\mathrm{S}}$

In Fig.6 we have shown how the scalar spectral index changes with the model parameter. We also see that spectral index is almost constant in both the large and small field sector of MHI. The current bound on $n_{S}$ from Planck 2015 has also been plotted.

The scale dependence of the spectral index itself is estimated from the scalar running and we have

$$
\begin{aligned}
n_{S}^{\prime} \simeq- & 2 \mathrm{M}_{\mathrm{P}}^{4} H^{\prime}(\phi) H^{\prime \prime \prime}(\phi) /\left.H^{2}(\phi)\right|_{\phi=\phi_{\mathrm{CMB}}} \\
& +\left.16 \epsilon_{H} \eta_{H}\right|_{\phi=\phi_{\mathrm{CMB}}}-\left.8 \epsilon_{H}^{2}\right|_{\phi=\phi_{\mathrm{CMB}}} \\
\simeq- & \frac{\mathrm{M}_{\mathrm{P}}^{4} \alpha^{4}}{2}\left[-32+30 \mathscr{L} W\left[\alpha, N_{\mathrm{CMB}}\right]\right. \\
& +33 \cosh \left(2 \cosh ^{-1} \mathscr{L} W\left[\alpha, N_{\mathrm{CMB}}\right]\right) \\
& \left.+\cosh \left(3 \cosh ^{-1} \mathscr{L} W\left[\alpha, N_{\mathrm{CMB}}\right]\right)\right] \\
& \times \mathscr{L} W\left[\alpha, N_{\mathrm{CMB}}\right]^{-4}\left(\mathscr{L} W\left[\alpha, N_{\mathrm{CMB}}\right]+1\right)^{-1} \\
& \times\left(\mathscr{L} W\left[\alpha, N_{\mathrm{CMB}}\right]-1\right)^{-2}
\end{aligned}
$$

Here in Fig. 7 logarithmic variation of the absolute value of scalar running with $\alpha$ has been plotted. From the figure 


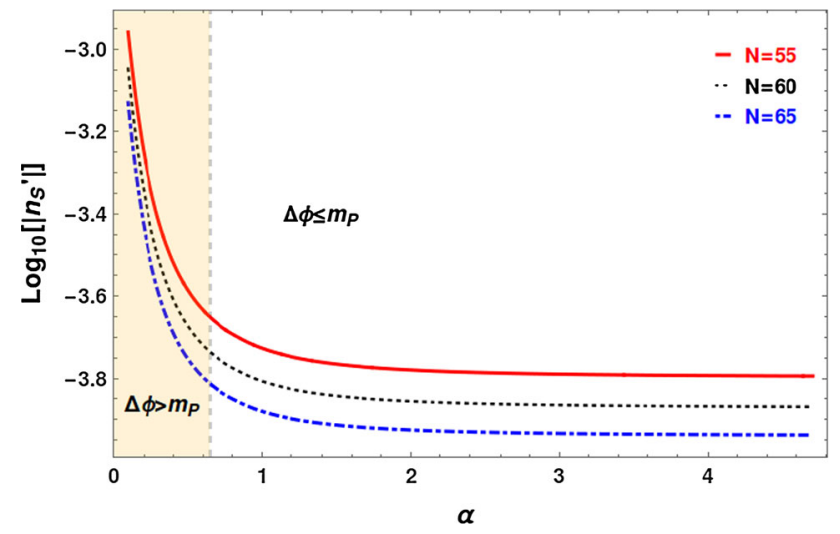

Fig. 7 The logarithmic variation of the absolute value of the scalar running with $\alpha$ for three different values of $N_{\mathrm{CMB}}$ has been plotted. The shaded vertical region is the result for large field sector of the model under consideration

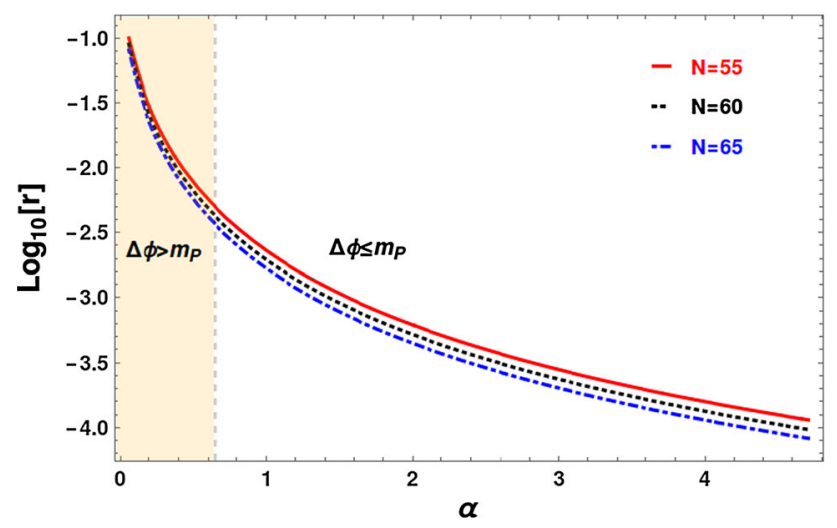

Fig. 8 Logarithmic variation of the tensor to scalar ratio with $\alpha$ for three different values of $N_{\mathrm{CMB}}$ has been plotted. The shaded vertical region corresponds to the large field sector of the MHI

it is clear that MHI predicts very small running of the spectral index. The maximum amount of scalar running that can achieved in MHI is $\left|n_{\mathrm{S}}^{\prime}\right| \sim 10^{-3}$.

Finally, the tensor to scalar ratio is found to have the following form

$$
\begin{aligned}
r & \left.\simeq 16 \epsilon_{H}\right|_{\phi=\phi_{\mathrm{CMB}}} \\
& \simeq 8 \mathrm{M}_{\mathrm{P}}^{2} \alpha^{2} \frac{\mathscr{L} \mathrm{W}\left[\alpha, \mathrm{N}_{\mathrm{CMB}}\right]+1}{\mathscr{L} \mathrm{W}\left[\alpha, \mathrm{N}_{\mathrm{CMB}}\right]^{2}\left(\mathscr{L} \mathrm{W}\left[\alpha, \mathrm{N}_{\mathrm{CMB}}\right]-1\right)}
\end{aligned}
$$

In Fig. 8 we have plotted the tensor to scalar ratio (in $\log _{10}$ scale) with $\alpha$ for three different values of $N_{\mathrm{CMB}}$. We see that MHI can address wide range of values of tensor to scalar ratio, $10^{-4} \lesssim r<10^{-1}$ depending on the model parameter $\alpha$. But $r \gtrsim 0.07$ has to be discarded which is observationally forbidden [34], which effectively determines a rough lower bound on the model parameters, $\alpha>0.094561$.

In Fig. 9 we have shown variation of the MHI energy scale with the tensor to scalar ratio. So in order to achieve $r \sim 0.07$ we need an energy scale $V_{0}^{\frac{1}{4}} \sim 1.5 \times 10^{16} \mathrm{GeV}$.

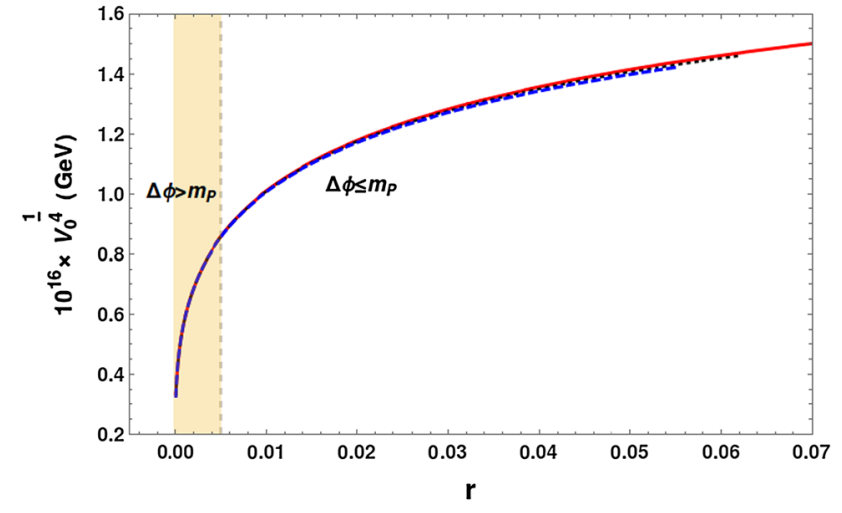

Fig. 9 Variation of the MHI energy scale with $r$ has been shown for three different values of $N_{\mathrm{CMB}}$. Red solid line is for $N_{\mathrm{CMB}}=55$, black dotted curve represents $N_{\mathrm{CMB}}=60$ and the blue dashed line for $N_{\mathrm{CMB}}=65$. The shaded vertical region represents small field sector of $\mathrm{MHI}$

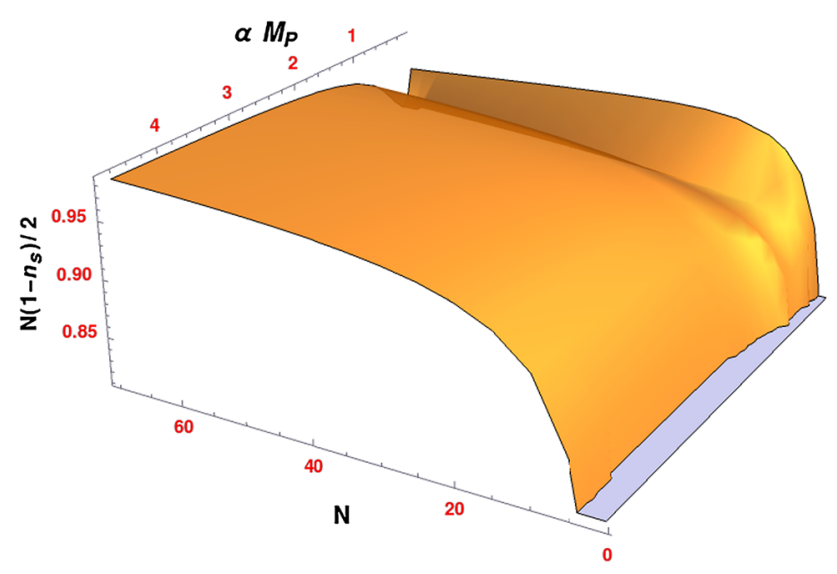

Fig. 10 The variation of $N\left(1-n_{S}\right) / 2$ in MHI with the model parameter $\alpha$ and the number of $e$-foldings, $\mathrm{N}$, has been shown

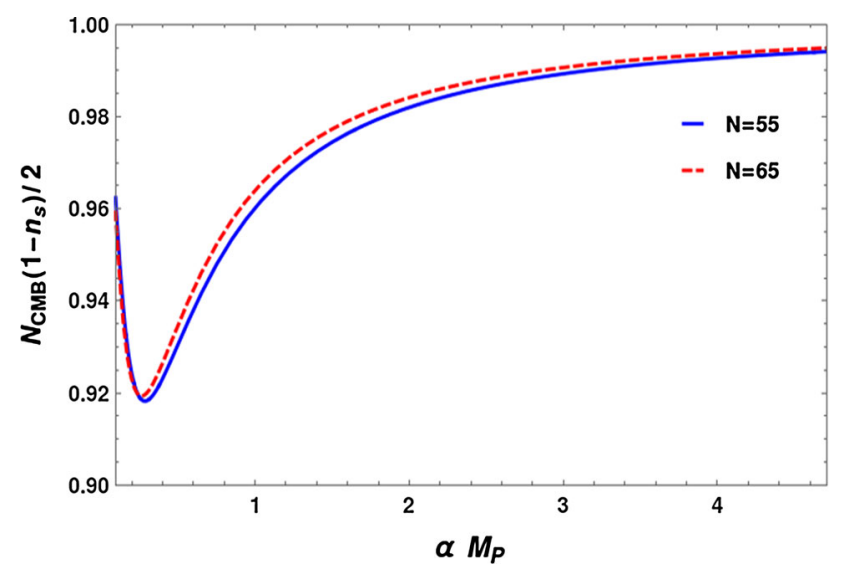

Fig. 11 Variation of $N_{\mathrm{CMB}}\left(1-n_{S}\right) / 2$ in MHI with $\alpha$ has been shown for two different values of $N_{\mathrm{CMB}}$. Blue solid line is for $N_{\mathrm{CMB}}=55$, red dotted curve represents $N_{\mathrm{CMB}}=65$ 
Fig. 12 Variation of $\alpha^{2} M_{P}^{2} r N^{2} / 8$ in MHI with the model parameter $\alpha$ and the number of $e$-foldings, $\mathrm{N}$, has been shown

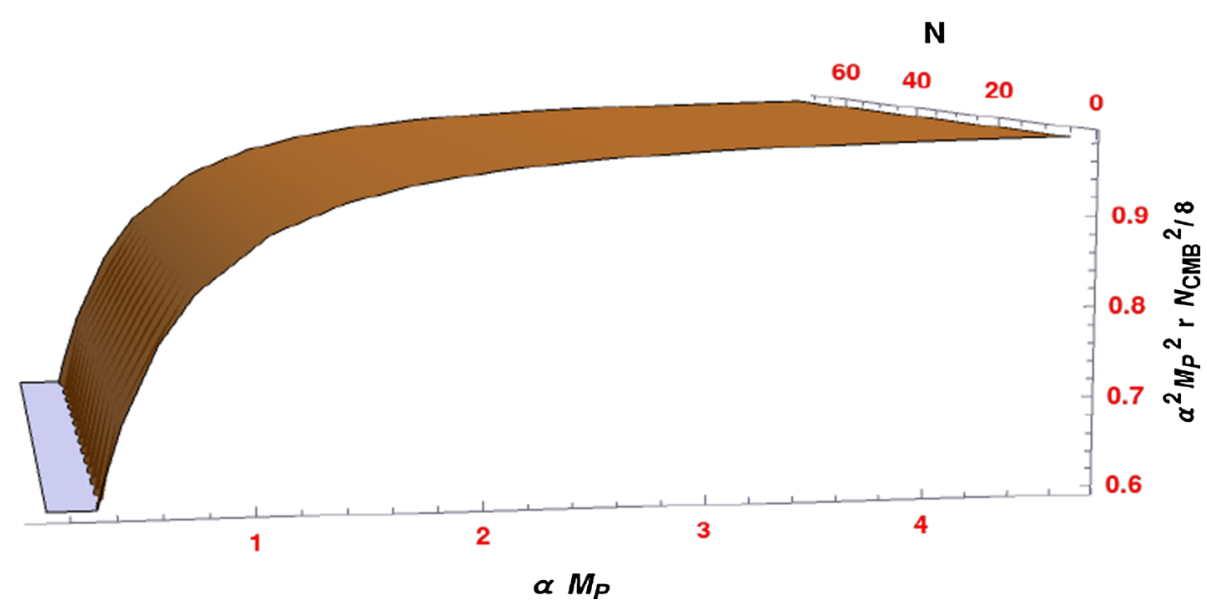

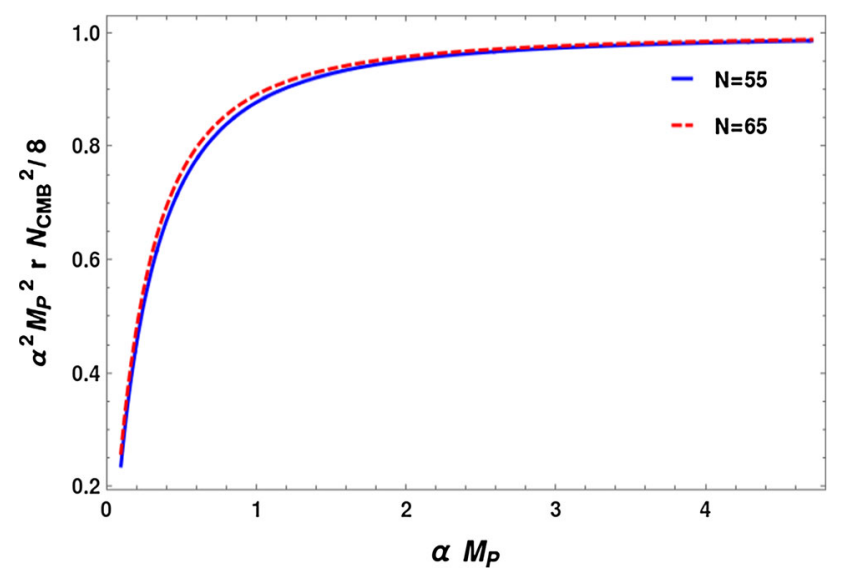

Fig. 13 Variation of $\alpha^{2} M_{P}^{2} r N_{\mathrm{CMB}}^{2} / 8$ in MHI with $\alpha$ has been shown for three different values of $N_{\mathrm{CMB}}$. Blue solid line is for $N_{\mathrm{CMB}}=55$, red dotted curve represents $N_{\mathrm{CMB}}=65$

\section{Similarity with $\alpha$-attractor class of inflationary models}

Now we shall show the resemblance of MHI with the $\alpha$ attractor class of models $[35,36]$ in the limit of $\alpha \phi \gg 1$. During inflation, within the slow-roll limit, the spectral index is given by

$$
\begin{aligned}
n_{S}-1 & \simeq-4 \epsilon_{H}+2 \eta_{H} \\
& \simeq-M_{P}^{2} \alpha^{2} \frac{3 \operatorname{sech}^{2}(\alpha \phi)-\operatorname{sech}^{3}(\alpha \phi)+2 \operatorname{sech}(\alpha \phi)}{1-\operatorname{sech}(\alpha \phi)} \\
& \approx-2 M_{P}^{2} \alpha^{2} \operatorname{sech}(\alpha \phi)
\end{aligned}
$$

where in the last step we have retained only the leading order term, keeping in mind that we are considering the limit $\alpha \phi \gg$ 1. Also, the scalar to tensor ratio, $r$, may be expressed as

$$
\begin{aligned}
r & \simeq 16 \epsilon_{H} \\
& \simeq 8 M_{P}^{2} \alpha^{2} \frac{\operatorname{sech}^{2}(\alpha \phi)\left[1-\operatorname{sech}^{2}(\alpha \phi)\right]}{(1-\operatorname{sech}(\alpha \phi))^{2}}
\end{aligned}
$$

$$
\approx 8 M_{P}^{2} \alpha^{2} \operatorname{sech}^{2}(\alpha \phi)
$$

where again in the last line we have kept leading order term in $\operatorname{sech}(\alpha \phi)$. Now the number of $e$-foldings, $N$, is approximately given by Eq. (16), which in the limit $\alpha \phi \gg 1$ may be written as

$N \approx M_{P}^{-2} \alpha^{-2} \cosh (\alpha \phi)$

In order to derive the above Eq. (29) we have made use of the fact that logarithmic function varies slowly and neglected the constant terms $\cosh \left(\alpha \phi_{\text {end }}\right)$ and $\ln \left[\cosh \left(\alpha \phi_{\text {end }} / 2\right)\right]$ during inflation. Combining Eqs. (27)-(29), we see that in the limit of $\alpha \phi \gg 1$

$n_{S}-1 \approx-\frac{2}{N} \quad$ and $\quad r \approx \frac{1}{M_{P}^{2} \alpha^{2}} \frac{8}{N^{2}}$

So from Eq. (30) we see that during inflation when $\alpha \phi \gg 1$, $\mathrm{MHI}$ indeed belongs to the class of $\alpha$-attractor models.

In Fig. 10 we have shown the variation of $N\left(1-n_{S}\right) / 2$ with the model parameter $\alpha$ and the number of $e$-foldings, $\mathrm{N}$. From the figure it is clear that for large values of the model parameter MHI belongs to the $\alpha$-attractor class of models and deviation occurs for the small values of the model parameter. In Fig. 11 we have shown the variation of $N_{\mathrm{CMB}}\left(1-n_{S}\right) / 2$ with the model parameter $\alpha$ for two different values of $N_{\mathrm{CMB}}$.

In Fig. 12 we have shown the variation of $\alpha^{2} M_{P}^{2} r N^{2} / 8$ with the model parameter $\alpha$ and the number of $e$-foldings, N. From the figure it is clear that for large values of the model parameter $\mathrm{MHI}$ indeed belongs to the $\alpha$-attractor class of models and small deviation occurs for the small values of the model parameter. In Fig. 13 we have plotted $\alpha^{2} M_{P}^{2} r N_{\mathrm{CMB}}^{2} / 8$ for two different values of $N_{\mathrm{CMB}}$.

So, from the above results it is quite transparent that $\mathrm{MHI}$ indeed falls into the category of $\alpha$-attractor class of inflationary models in the limit of $\alpha \phi \gg 1$. 


\section{Conclusion}

In this article we have revisited mutated hilltop inflation driven by a hyperbolic potential. Employing HamiltonJacobi formulation we found that inflation ends naturally through the violation slow-roll approximation. More interestingly, MHI has two different branches of inflationary solution. One corresponds to large field variation and the other represents small change in inflaton during the observable inflation depending on the model parameter.

Observable parameters as derived from this model are in tune with the latest observations for a wide range of the model parameter, $\alpha \mathrm{M}_{\mathrm{P}} \gtrsim 0.094561$. The scalar spectral index is found to be independent of the model parameter with a small negative running. We have also found that MHI can address a broad range of the tensor to scalar ratio, $0.0001 \lesssim r<$ 0.07. In a nutshell, MHI though does not belong to the usual hilltop inflation is extremely attractive with only one model parameter consistent with recent observations. Not only that, in the limit of $\alpha \phi \gg 1$, MHI closely resembles the $\alpha$-attractor class of inflationary models.

Acknowledgements I would like to thank Supratik Pal for useful discussions and constructive suggestions. I would also like to thank IUCAA, Pune for giving me the opportunity to carry on research work through their Associateship Program. Finally author would like to sincerely thank anonymous reviewers for their critical and constructive suggestions on the first version of this work.

Open Access This article is distributed under the terms of the Creative Commons Attribution 4.0 International License (http://creativecomm ons.org/licenses/by/4.0/), which permits unrestricted use, distribution, and reproduction in any medium, provided you give appropriate credit to the original author(s) and the source, provide a link to the Creative Commons license, and indicate if changes were made. Funded by SCOAP ${ }^{3}$.

\section{References}

1. A.A. Starobinsky, A new type of isotropic cosmological models without singularity. PLB 91(1), 99-102 (1980)

2. K. Sato, First-order phase transition of a vacuum and the expansion of the universe. MNRAS 195(3), 467-479 (1981)

3. A.H. Guth, The inflationary universe: a possible solution to the horizon and flatness problems. PRD 23, 247 (1981)

4. A. Albrecht, P.J. Steinhardt, Cosmology for grand unified theories with radiatively induced symmetry breaking. PRL 48, 1220 (1982)

5. A.D. Linde, A new inflationary universe scenario: s possible solution of the horizon, flatness, homogeneity, isotropy and pimordial monopole problems. PLB 108, 389 (1982)

6. A.A. Starobinsky, Relict gravitation radiation spectrum and initial state of the universe. JETP lett 30(682-685), 131-132 (1979)

7. V.F. Mukhanov, G.V. Chibisov, Quantum fluctuations and a nonsingular universe. JETP Lett. 33, 532-535 (1981)

8. S.W. Hawking, The development of irregularities in a single bubble inflationary universe. PLB 115(4), 295-297 (1982)
9. A.A. Starobinsky, Dynamics of phase transition in the new inflationary universe scenario and generation of perturbations. PLB 117(3-4), 175-178 (1982)

10. A.H. Guth, S.Y. Pi, Fluctuations in the new inflationary universe. PRL 49(15), 1110 (1982)

11. V.F. Mukhanov, H.A. Feldman, R.H. Brandenberger, Theory of cosmological perturbations. Phys. Rep. 215, 203 (1992)

12. E.D. Stewart, D.H. Lyth, A more accurate analytic calculation of the spectrum of cosmological perturbations produced during inflation. PLB 302, 171 (1993)

13. G.F. Smoot et al., Structure in the COBE differential microwave radiometer first-year maps. ApJ 396, L1-L5 (1992)

14. G. Hinshaw et al., Nine-Year Wilkinson Microwave Anisotropy Probe (WMAP) observations: cosmological parameter results. ApJ Suppl. Ser. 208, 19 (2013)

15. P.A.R. Ade et al., Planck 2015 results-XIII. Cosmological parameters. A\&A 594, A13 (2016)

16. P.A.R. Ade et al., Planck 2015 results-XX. Constraints on inflation. A\&A 594, A20 (2016)

17. J. Martin, C. Ringeval, V. Vennin, Encyclopædia inflationaris. Phys. Dark Universe 5, 75 (2014)

18. J. Martin, C. Ringeval, R. Trotta, V. Vennin, The best inflationary models after Planck. JCAP 03, 039 (2014)

19. B.P. Abbott et al., Observation of gravitational waves from a binary black hole merger. PRL 116, 061102 (2016)

20. B.P. Abbott et al., Observation of gravitational waves from a 22solar-mass binary black hole coalescence. PRL 116, 241103 (2016)

21. K.N. Abazajian et al. CMB-S4 Science Book. (2016). arXiv: 1610.02743 (arXiv preprint)

22. A.A. Starobinskii, On a nonsingular isotropic cosmological model. Sov. Astron. Lett 4, 155-159 (1978)

23. A.R. Liddle, P. Parsons, J.D. Barrow, Formalising the slow-roll approximation in inflation. PRD 50, 7222-7232 (1994)

24. W. Kinney, Hamilton-Jacobi approach to non-slow-roll inflation. PRD 56, 2002-2009 (1997)

25. D. Salopek, J. Bond, Nonlinear evolution of long wavelength metric fluctuations in inflationary models. PRD 42, 3936-3962 (1990)

26. A. Muslimov, On the scalar field dynamics in a spatially flat Friedman universe. CQG 7, 231 (1990)

27. H. Motohashi, A.A. Starobinsky, J. Yokoyama, Inflation with a constant rate of roll. JCAP 2015(09), 018 (2015)

28. H. Motohashi, A.A. Starobinsky, Constant-roll inflation: confrontation with recent observational data. EPL 117(3), 39001 (2017)

29. H. Motohashi, A.A. Starobinsky, f (r) constant-roll inflation. EPJC 77(8), 538 (2017)

30. B.K. Pal, S. Pal, B. Basu, Mutated hilltop inflation: a natural choice for early universe. JCAP 01, 029 (2010)

31. B.K. Pal, S. Pal, B. Basu, A semi-analytical approach to perturbations in mutated hilltop inflation. IJMPD 21, 1250017 (2012)

32. L. Boubekeur, D.H. Lyth, Hilltop inflation. JCAP 07, 010 (2005)

33. K. Kohri, C.M. Lin, D.H. Lyth, More hilltop inflation models. JCAP 12, 004 (2007)

34. P.A.R. Ade et al., Improved constraints on cosmology and foregrounds from bicep2 and keck array cosmic microwave background data with inclusion of $95 \mathrm{ghz}$ band. PRL 116, 031302 (2016)

35. R. Kallosh, A. Linde, Universality class in conformal inflation. JCAP 2013(07), 002 (2013)

36. M. Galante, R. Kallosh, A. Linde, D. Roest, Unity of cosmological inflation attractors. PRL 114(14), 141302 (2015)

37. B.K. Pal, S. Pal, B. Basu, Confronting quasi-exponential inflation with WMAP seven. JCAP 04, 009 (2012)

38. D.H. Lyth, What would we learn by detecting a gravitational wave signal in the cosmic microwave background anisotropy? PRL 78, 1861 (1997) 